\section{LA EXPLORACIÓN NATURALISTA DE MADEIRA EN EL SIGLO XIX: LOS VIAJEROS ALEMANES Y SU INTERÉS POR ESTA ISLA*}

\author{
Sandra Rebok \\ Área de Cultura Científica \\ CSIC, Madrid
}

\section{THE SCIENTIFIC EXPLORATION OF MADEIRA IN THE 19TH CENTURY: GERMAN TRAVELLERS AND THEIR INTEREST IN THE ISLAND}

\begin{abstract}
This essay aims to describe the various German scientific travellers who visited the island of Madeira from the second half of the 19th century onwards, the studies carried out by them, and the publications which resulted from their work. It documents the beginning of the systematic scientific research of this island, analyzing the focus of this interest as well as the form in which it developed in the decades to follow. After a general introduction about the genre of scientific travel literature, the article presents the significance of Madeira for German natural scientists and physicians of the 19th century, their impressions about the island, as well as the manifold information they offer about the geography, botany, landscapes, commerce, agriculture, and habits of the populations and their daily life. In this context, the views of Madeira expressed by these travellers will be of interest, particularly in the context of the differences they observed between Madeira and the Canary Islands.
\end{abstract}

KEY WORDS: Scientific travellers; expeditions; Madeira; History of Science; Canary Islands.

\section{INTRODUCCIÓN}

Aunque ya visitada con anterioridad por numerosos viajeros europeos' ${ }^{1}$, no sería hasta la segunda mitad del siglo XIX cuando un mayor número de viajeros de habla alemana ${ }^{2}$ descubrieran la isla de Madeira para sus fines, ya fueran estos las curiosidades de los viajeros en general, o la de los científicos en particular. Serian varios factores los que llamasen su atención: el fundamento para su estatus especial era sin duda su situación como isla; es decir, estar alejada del continente, y el hecho de formar parte de Portugal, sin ser una colonia, lo cual constituyó una fuente de frecuentes comparaciones con las Islas Canarias. Además, ya en la primera mitad del siglo XIX Madeira se veía como un puente entre el confort europeo y el lujo tropical,
RESUMEN: Este estudio tiene como objetivo dar a conocer a diferentes viajeros científicos de origen alemán que visitaron Madeira a partir de mediados del siglo XIX, los estudios realizados por ellos así como las publicaciones que resultaron de ello. Se trata principalmente de documentar los inicios de la investigación científica sistemática en la isla, analizar este interés y la forma en que se desarrolló en las décadas siguientes. Tras una introducción general sobre el género de la literatura de viajes, el enfoque se centra en el significado de esta isla para los visitantes, naturalistas y médicos alemanes del siglo XIX, las propias impresiones de las regiones recorridas, así como la variada información ofrecida sobre su geografía, botánica, los distintos paisajes, el comercio, la agricultura, las costumbres de la población y su vida cotidiana. Se analizará especialmente la mirada de estos viajeros sobre Madeira y por otro lado se realizará una comparativa que permita conocer las diferencias que se percibieron entre Madeira y las Islas Canarias.

PALABRAS CLAVE: Viajeros científicos; expediciones; Madeira; Historia de la Ciencia; Islas Canarias.

un "Jardin del Edén", tal como la denominaba una guía de viajes inglesa ${ }^{3}$, que también sirvió de referencia a los viajeros alemanes.

Este estudio tiene como objetivo dar a conocer diferentes viajeros alemanes que visitaron Madeira a partir de mediados del siglo XIX, los estudios realizados por ellos así como las publicaciones que resultaron de ello. Se trata principalmente de documentar los inicios de la investigación científica sistemática en la isla, determinar la composición de este interés y la forma en que se desarrolló en las décadas a seguir. Adicionalmente, para completar esta presentación también se tomarán en cuenta meros viajeros típicamente románticos $u$ otro tipo de estancias en la isla. A veces resultaría incluso difícil la diferenciación 
entre trabajos científicos y narraciones de viaje, debido al carácter de dichas publicaciones, que mezclan ambos tipos de información.

Al trabajar con el género de literatura de viajes hay que ser consciente del gran abanico de diferentes enfoques que este término abarca. Debido a esta heterogeneidad y a la multidimensionalidad de este concepto, tampoco la categoría viajes científicos se deja delimitar tan claramente: por un lado se aproxima a los meros viajeros con cierto interés general por el país visitado; por el otro, a los científicos residentes durante muchos años en un país ajeno, sin llevar a cabo viajes propiamente dichos, o bien los estudios científicos realizados a partir de documentos 0 datos recibidos, sin conocer personalmente la región descrita. Otra categoría cercana son las guias de viajes, que también aportan una gran cantidad de conocimiento científico sobre un país, sin que hayan sido resultado de una investigación propia. La delimitación entre estos enfoques es algo difusa y a menudo el título de la publicación tampoco contribuye a una aclaración en este sentido.

Otro factor a tener en consideración es la evolución del enfoque científico que aplican los viajeros a lo largo del siglo XIX: mientras a principios de este siglo prevalecen los estudios holísticos que tratan de representar la realidad ajena en sus más diversas facetas, sin profundizar en cuestiones concretas, en la segunda mitad del siglo XIX, con el desarrollo de las disciplinas científicas, los estudios realizados llegan a ser más precisos, con un objetivo concreto y claramente definido. Ya no abarcan un conjunto de facetas distintas, sino que se limitan a unas regiones y un campo científico determinado. A medida que se amplía la investigación científica, los viajeros consultan las obras publicadas anteriormente que abarcan los mismos aspectos y en sus propias publicaciones hacen referencias explícitas a estos antecedentes e incluso a veces desvelan su intención de continuar, profundizar o rectificar estos estudios anteriores.

Al estudiar los relatos científicos la duración de la estancia de investigación supone un criterio relevante. Otro aspecto importante es el motivo del viaje de investigación, es decir, si éste era meramente científico o si estaba vinculado con otros intereses, que podrian ser de tipo económico o turístico. Muy unido con esta cuestión se encuentra el tema de la financiación del viaje, que influia tanto en el objetivo de la investigación como en el enfoque de la posterior publicación. Y finalmente, también entre los viajeros científicos se manifiestan posturas muy diversas hacia la cultura ajena, que indudablemente tuvieron su influencia en la orientación de su publicación. Todo esto se hace más visible en la parte narrativa que ofrecen muchas de estas obras, o en los relatos de viajes que algunos de estos autores publicaron al margen de sus aportaciones científicas.

Debido a las mencionadas difusas delimitaciones del género literatura científica de viajes, es preciso definir lo que se entiende aquí por un relato de viaje científico en su sentido más estricto. Según la definición aplicada, entran en primer lugar aquellos estudios que se centran en generar, comprobar, recopilar o profundizar un cierto conocimiento durante un viaje, es decir, aquellos estudios que se basan en tomar mediciones, además de encontrar, clasificar y representar gráficamente los nuevos objetos encontrados, así como los que se dedican a establecer estudios comparados, pero también se toman en consideración descripciones con un enfoque científico general.

Los trabajos incluidos en esta investigación además fueron seleccionados por diferentes criterios para asegurar en la medida de lo posible su orientación científica. Estos criterios se refieren en primer lugar a factores tales como el hecho de si el autor había realizado un estudio del estado de la investigación hasta este momento; la existencia de notas a pie de página con referencias a otros trabajos; la existencia de una lista con la bibliografía utilizada; una clara estructura del trabajo presentado; así como la presentación evidente de los resultados, sea en forma de texto, de tablas, de catálogos o de representaciones gráficas. Esto se refiere tanto a las investigaciones en el campo de las Ciencias Naturales, como a los estudios sobre la Historia del Arte y otro tipo de trabajo histórico llevado a cabo en los archivos. De ello resulta la necesidad de que las publicaciones fruto de dichos viajes de exploración deban ser investigadas también desde el enfoque de la Historia de la Ciencia. Esta interdisciplinariedad en el estudio de la literatura de viaje ayuda por un lado a reconstruir esta faceta de los viajes realizados por esta isla, y por otro contribuye con un material sumamente interesante a reconstruir la historia de la investigación naturalista de Madeira y, no en último lugar, al estudio del desarrollo de las disciplinas académicas. 
El estudio presentado se basa en textos en los que los autores describieron detalladamente su periplo por Madeira, a menudo realizado como parte de su viaje por las Islas Canarias, por el sur de Europa, o incluso como escala de un más largo viaje hacia las Américas. El enfoque se centra en el significado de esta isla para sus visitantes, naturalistas y médicos alemanes del siglo XIX, las propias impresiones de las regiones recorridas, así como la variada información ofrecida sobre su geografía, botánica, los distintos paisajes, el comercio, la agricultura, las costumbres de la población y su vida cotidiana. Se analizará especialmente la mirada de estos viajeros a Madeira en general y las diferencias que entre Madeira y las Islas Canarias se percibieron en particular. Otra pregunta que se tiene en cuenta en este trabajo es qué representó para estos personajes, fueran científicos o no, el hecho de que se tratase de una isla.

Debido a la escasa literatura secundaria existente sobre este tema ${ }^{4}$, se trata éste de un trabajo introductorio que ofrece una visión general, lejos de poder ser exhaustivo en su temática, con un carácter más descriptivo que analítico, a fin de motivar el ulterior estudio de diferentes cuestiones ${ }^{5}$ que surgirán a continuación.

\section{Estudios realizados sobre Madeira: Visión general}

Entre los primeros trabajos sobre Madeira se encuentran diversas publicaciones que se dedicaron al estudio de su clima, debido a la importancia que tenía la isla en este tiempo como balneario, sobre todo para los enfermos de tuberculosis. Una obra citada a menudo en este contexto es la de Karl Mittermeier, publicada por primera vez en 1855 , de la que más tarde, en $1885^{6}$, surgiría una segunda edición modificada y considerablemente ampliada. Se trata de un análisis cientifico sobre las saludables condiciones climáticas que favorecian su establecimiento como lugar de curación.

Rudolph Schultz, viajero alemán que continuó esta línea de investigación abordando la enfermedad de la tuberculosis, dio lugar a una publicación sobre el tema en 18647 . Basada en una observación durante tres años, en la que Schultz visitó una parte sustancial de la isla ${ }^{8}$, esta obra describe la importancia del clima en general para el organismo humano, sobre todo en relación con sus características sanitarias en el proceso de curación de enfermedades.
Debido al clima suave y a la cercanía tanto del mar como de la sierra, que evita así largas caminatas para el enfermo, considera que Madeira, y particularmente su capital Funchal, ofrece las condiciones óptimas para el tratamiento de la tuberculosis ${ }^{9}$. Se trata de un estudio bien documentado, que ofrece una amplia bibliografia ${ }^{10}$, y cuyos dos últimos capítulos se dedican a la descripción del tipo de enfermedades que se daban entre los habitantes de Madeira, así como a la comprobación de los resultados de las curas alli practicadas. Finalmente, también brinda información práctica para la realización de viajes a esta isla".

Otro estudio climático de la isla de Madeira, y en especial de su capital, es la tesis doctoral del médico Ferdinand Christmann, del año $1889^{12}$. Debido a su carácter científico, está basado en la revisión de los trabajos efectuados anteriormente sobre esta materia ${ }^{13}$. El autor lamenta que en Alemania esta isla no haya alcanzado el mismo renombre como sanatorio para enfermos de pulmón que el adquirido en otros paises europeos como Francia y Gran Bretaña ${ }^{14}$. A fin de promover este proceso de concienciación entre sus conciudadanos, destaca las ventajas que, debido a su clima, la ciudad de Funchal aporta a estos enfermos, ofreciendo detalladas explicaciones apoyadas en tablas con mediciones exactas de la temperatura, la humedad del aire, la cantidad de lluvia, la presión atmosférica, los vientos, las nubes y la cantidad de ozono, para, finalmente, incorporar datos sobre el tipo de enfermedad que más fácilmente podría alcanzar su curación en la isla, así como el tiempo estimado para ello, en contraposición a aquellas frente a las que no se deberian albergar grandes esperanzas.

Otro aspecto de carácter médico que ha sido investigado en relación con Madeira ha sido la enfermedad de la lepra $^{15}$; asimismo, pudo localizarse un trabajo que mostraba que el clima de esta isla había sido objeto de estudio también en lo que respecta a la agricultura ${ }^{16}$.

En cierto sentido, y en relación con el interés por el clima de Madeira, puede ser vista la preocupación por la vegetación de esta isla, surgida ya en un momento anterior. En 1859 Hermann Schacht publicó un libro ${ }^{17}$, redactado como un informe para el Ministerio Real Prusiano para asuntos de agricultura, en el que proporcionaba una breve descripción de su viaje y estancia en las islas de Madeira y Tenerife, con una muy detallada información sobre la flora de dichos lugares. Este viaje fue financiado por dis- 
tintos Ministerios Reales así como por la Real Academia de Ciencias y su enfoque científico estaba dirigido a las plantas útiles de ambas islas, a fin de intentar conocer sus particularidades y métodos de cultivo. Se trataba de una estancia de casi dos años para ambas islas, lo que le permitió una más profunda dedicación a las cuestiones investigadas. Respecto a los resultados, el autor expresó explícitamente el deseo de que no sirvieran solamente al interés de la ciencia en general, sino que también brindase información de valor para la agricultura.

De esta manera, las primeras 16 páginas ofrecen una visión general de Madeira y Tenerife, tratando los más diversos aspectos; a continuación se presenta una descripción de carácter científico de las plantas encontradas, siempre con una visión comparativa respecto a las dos islas. No obstante, aunque en el apéndice añade una parte narrativa en la que describe el viaje propiamente dicho, el resto del libro mantiene la estructura de un tratado científico.

Lo que llamó particularmente su atención fueron las distintas plantas que descubrió según la altura; es decir, las zonas de cultivo, la diferencia observada entre plantas de carácter más tropical de la parte del sur, en comparación con las de la región septentrional, así como el suelo volcánico de la isla. En relación con la diferencia entre Madeira y Tenerife menciona como una gran ventaja de la primera ${ }^{18}$ su riqueza en recursos acuíferos. Aunque solo ubicada de tres a cinco grados de latitud más al sur de Madeira, la flora de Tenerife se revelaba muy distinta, ya que según sus observaciones, Tenerife poseía tanto plantas autóctonas de las Canarias, como otras procedentes del continente africano ${ }^{19}$. El clima de ambas islas lo veía bastante similar pero, según él, debido a un menor régimen de precipitaciones durante los meses de invierno, Santa Cruz de Tenerife y Las Palmas de Gran Canaria serían mucho más aptas para enfermos necesitados de un clima seco. Sin embargo, en estas últimas islas no se daba la infraestructura necesaria, mientras que Funchal ofrecía todas las comodidades y el lujo de Inglaterra ${ }^{20}$. Otras diferencias se apreciaban en el hecho de que Madeira no se hallaba habitada en el momento en que fue descubierta y en que todos los animales domésticos fueron importados, sobre todo desde Inglaterra y Portugal, mientras que las Islas Canarias ya eran cultivadas por los guanches en el momento de la conquista castellana, habiendo alcanzado un tamaño considerable su agricultura y ganadería.
También Oswald Heer se ocupó de la vegetación de esta isla, con las diferencias apreciables de que, en lugar de una comparación con Tenerife, se limitó a la región de San Jorge, y el hecho de que centrase sus esfuerzos en definir la vegetación autóctona de la isla ${ }^{21}$. Durante su viaje en el año 1851 estudió las condiciones geológicas e intentó definir lo que eran las especies originales de la isla en comparación con las importadas a lo largo del tiempo. En relación con esta cuestión presentaba un gran significado su estatus como isla por un lado, y su conexión con el continente europeo, por el otro. La ventaja que veía en Madeira era el hecho de que la isla ofrecía muchas pendientes de rocas aisladas con difícil acceso, donde la vegetación original se podía conservar mejor. Se trata de un libro de carácter científico, bien documentado, con numerosas referencias a trabajos realizados en este campo anteriormente ${ }^{22}$.

Inspirado por el Prof. Heer, y continuando su trabajo, a lo largo de varios viajes, también Georg Hartung se dedicó al estudio de aspectos geológicos de las islas Madeira y Porto Santo ${ }^{23}$. El resultado fue plasmado en una obra muy detallada y con gran rigor científico, en la que la primera parte aborda la situación geológica, y la segunda, redactada por Karl Meyer, la condición paleológica, con un índice sistemático de los restos fósiles de estas islas ${ }^{24}$.

Por su parte, Richard Greeff constituye un ejemplo más de científico dedicado a la investigación naturalista y principalmente zoológica de Madeira. Al principio del estudio de estas cuestiones ${ }^{25}$, basadas en su viaje de 1866 a 1867, explica detalladamente los motivos por los que consideraba esta isla tan interesante en este sentido, motivos que por su relevancia para el tema de islas y ciencia serán presentados a continuación.

Greeff sostenía que una de las tareas más interesantes y fructiferas de la ciencia era la investigación naturalista de regiones apartadas y encerradas en sí mismas, sobre todo en lo que relativo a vegetación y zoología. El lugar ideal para estudiar tales regiones en su aislamiento se encuentra de una manera más perfecta y pura en las islas pequeñas, situadas lejos del continente. En función de las características particulares de la ubicación y naturaleza de las islas, en éstas resaltan los tres factores básicos que intervienen en la formación de los cuerpos orgánicos de la naturaleza (el clima, los nutrientes y la composición del 
suelo), pudiendo ser más fácilmente definidos que en tierra firme. Además, esta situación geográfica caracterizada por el aislamiento dificulta considerablemente la inmigración de plantas o animales del continente o de otras islas lejanas dando lugar a un desarrollo más uniforme de las formas de vida alli establecidas, bajo las condiciones dadas, sin perturbaciones ni influencias externas. En los casos donde se ha producido una inmigración, normalmente es posible averiguar por qué partes y vías ha tenido lugar, de qué regiones exactamente procede, y se permite analizar la modificación por adaptación y cruzamiento que han experimentado estas especies nuevas.

Según Greeff, las experiencias más valiosas sobre el origen y la repercusión de estas conclusiones resultan de una observación comparada de las especies autóctonas de estas islas con las que inmigraron más tarde, o bien de las formas de vida que pertenecen a las mismas especies, pero que se desarrollan en otras regiones bajo condiciones distintas. Por lo tanto, aquí se podía más fácil y exactamente investigar las condiciones que influyeron en la vida de las plantas y los animales, y los efectos que estas circunstancias ejercían a largo plazo sobre dichas formas de vida. Como consecuencia, este estado de isla ofrece un excelente material para la teoría de la evolución, cuyo desarrollo y profundización consideraba una de las tareas más importantes de las ciencias naturales, ya que aportaba significativas explicaciones sobre el origen de las especies en los reinos animal y vegetal. Por todo esto, el autor dedicó su esfuerzo científico a la investigación de las Islas Canarias y de Madeira, bajo los criterios mencionados.

Además, en su opinión, pocos lugares en el mundo podian ofrecer, en un espacio tan limitado, tantas características científicas particulares, razón por la cual estas islas merecían en grado sumo la atención de los científicos. Las sierras volcánicas, que emergen casi directamente del océano, ofrecen un campo de trabajo excelente para el geólogo, ya que le ofrecen datos sobre su historia y su origen. Gracias a su gran riqueza en caracteristicas raras e interesantes han contribuido a resolver importantes cuestiones sobre el origen y la constitución de los volcanes. No menos interesante resultaria el mundo vegetal, que se presenta en sucesivos niveles desde el mar hasta las cimas más altas. Cada nivel ofrece una zona donde la flora es distinta de la anterior, encontrándose en el norte una vegetación diferente de la del sur. Finalmente, también el mundo animal, aunque a primera vista pueda parecer más bien pobre, viene a ser muy interesante y rico ${ }^{26}$, por lo que el autor ofrece a continuación una comparación entre la fauna de Canarias y la de Madeira ${ }^{27}$.

Aparte de este tratado científico, Greeff también publicó otro libro sobre este viaje, que fue estructurado siguiendo más el estilo de una narración de viaje, enriquecida, eso sí, con profusión de datos y referencias ${ }^{28}$. Este relato está basado en las cartas redactadas durante el viaje que desde Londres le llevaría, además de a Madeira, a las Islas Canarias, Portugal, Marruecos y España. En él narra sus vivencias personales durante los desplazamientos efectuados desde Funchal al interior de la isla. El objetivo de este trabajo consistía en hacer llegar a un amplio público y sin conocimentos especializados información de carácter general no incluida anteriormente en su estudio científico.

Otro trabajo que contribuyó notablemente a la investigación geológica de la isla fue el realizado por Karl Wilhelm Fritsch ${ }^{29}$. Como objetivo inicial de su viaje de exploración entre los años 1862 a 1863 indica la investigación geológica del archipiélago de las Islas de Cabo Verde. En el camino de ida tenía previsto visitar Madeira y algunas de las Islas Canarias, por una parte para adaptarse más fácilmente al clima del archipiélago de Cabo Verde y, por otra, para llevar a cabo en estas islas más conocidas estudios preparatorios prácticos a fin de conectarlos con los anteriores, de tipo teórico. Sin embargo, la epidemia de fiebre amarilla que se desarrolló en Santa Cruz de Tenerife en 1862 le mantuvo durante todo el invierno en las Canarias, de manera que no llegaría a alcanzar el destino inicial de su viaje. El estilo que el autor mantiene en este trabajo constituye una mezcla entre narración de viaje y guía de referencia sobre Madeira y las Islas Canarias, junto a resultados de su propia investigación. A final, proporciona noticias generales sobre Madeira, compuestas a partir de otros trabajos, y las contrasta con sus comentarios o impresiones personales.

Alphons Stübel fue otro científico, también con un marcado interés geológico, que descubrió el significado de Madeira en este contexto. A finales de 1862 llegó a Madeira, pero ya un mes más tarde debía partir para Canarias, para después dirigirse a Cabo Verde. Siguiendo su investigación en estas últimas islas, reconoció la gran importancia de Madeira para la teoría volcánica. Por lo tanto, se decidió a emprender otra estancia más larga en esta isla en sep- 
tiembre de 1863, para allí continuar su trabajo volcánico y llevar a cabo una exhaustiva investigación geológica del suelo de Madeira. Sin embargo, tras su regreso a Alemania en el verano de 1865, dejó a lado este estudio, y junto a Karl von Fritsch y Wilhelm Reiss se dedicó a otras tareas. A excepción de unos breves trabajos de Stübel sobre Madeira ${ }^{30}$, el material traído desde esta isla quedó inédito hasta que Walter Bergt encontró valiosos manuscritos en su legado que decidió publicar ${ }^{31}$ debido al hecho de que Madeira había tenido un gran significado en el desarrollo de las ideas de Stübel ${ }^{32}$. Se trata de un trabajo netamente científico, y muy centrado en la geología, ya que aparentemente ni siquiera la vegetación llamó la atención de este investigador ${ }^{33}$. Al final de su obra se añadió un índice de todos los mapas e ilustraciones de Madeira que se encontraban en el Museum für Länderkunde en Leipzig, algunos de los cuales se incluyeron en este trabajo.

Al margen de estos visitantes, motivados por intereses y objetivos científicos, se dieron asimismo los que meramente se acercaban atraídos por el mero placer del encuentro con lo exótico, envueltos en un halo de postromanticismo. $A$ veces, estos viajeros sintieron también cierto interés por los estudios científicos realizados en esta región, habiendo leído a menudo estas obras, pero sin que ello les llevase a la realización de estudios propios. Con frecuencia, su estancia en Madeira ha sido solamente una escala de un periplo más amplio, y han influido más sus intereses personales por conocer estas tierras ajenas. Algunos de ellos también se dejan atribuir a los famosos viajes románticos, para los que Madeira también tuvo un significado particular por estar ubicada de manera aislada, lejos del continente.

Un ejemplo temprano de ello es la edición de las cartas del viaje que realizó el médico austriaco Augustin Trogher por una gran parte del sur de Europa ${ }^{34}$. Una de estas cartas se dedica a reunir sus impresiones sobre Madeira ${ }^{35}$, donde muchos aspectos le parecian "particulares" o "característi$\cos ^{\prime \prime 36}$. Por otra parte, según él, la isla poseía un doble significado como lugar de curación, no sólo para los enfermos de pulmón sino también para los de corazón, aunque en este caso no precisamente en el sentido fisiológico ${ }^{37}$.

Pocos años después se publicó el trabajo de Jegór Sivers sobre su viaje de Madeira a las Antillas y América Central ${ }^{38}$. Aquí se mantiene una mezcla de ciertos criterios científicos como son un índice de personas o lugares, una extensa bibliografia ${ }^{39}$, etc., con una descripción superficial del viaje, haciendo particular hincapié en sus aventuras. A pesar del hecho de que el propio título del trabajo anuncia aspectos memorables de su viaje y su investigación, se trata más bien de una repetición de determinados estereotipos sobre esta isla, en la que además se plasma cierta tendencia romántica; por ejemplo, cuando cita o compone poemas propios, y cuando ofrece sus reflexiones sobre "aquel paraíso, el más bello que he soñado" ${ }^{40}$, idealizando la naturaleza ${ }^{41}$.

Un estilo parecido, aunque no tan poético, se encuentra en el relato de Johannes Stenzel sobre su viaje por Madeira y las Islas Canarias ${ }^{42}$. De nuevo se trata de una descripción superficial del viaje, en la que la información sobre la primera isla solamente forma una parte pequeña ofrecida en este libro ${ }^{43}$.

Como consecuencia del creciente número de viajeros o científicos de habla alemana que a lo largo de la segunda mitad del siglo visitaron la isla, se veía la necesidad de ofrecerles informaciones útiles para su viaje o estancia. Una de las primeras obras que se entendió explícitamente como guía de Madeira, y que ha podido ser localizada, es la de Paul Langerhans, del año $1885^{44}$. Debido a la falta de manuales sobre esta isla con informaciones actualizadas y en idioma alemán, Langerhans editó una versión alemana del libro de Yate Johnson de $1857^{45}$, del cual tantas referencias se pueden hallar. Como aclara Langerhans, unos capitulos fueron redactados de nuevo, otros modificados y otros, simplemente traducidos, todo con la aprobación del autor inglés. Debido a su carácter de guía de viaje, se limita a dar todo tipo de información sobre la mejor época para viajar, precios, hoteles, etc., que pueda resultar útil para el viajero. En esta descripción general ofrece sus impresiones personales, que no estaban basadas en ningún estudio, pero trata muchos aspectos distintos y también añade conocimiento específico, aunque siempre con vista a su utilidad para el viajero. Además, ofrece numerosas referencias a trabajos científicos ${ }^{46}$.

Otro manual que ha podido ser consultado es el de Ronald Krohn, del año $1906^{47}$, que al igual que el anterior, también está basado en obras inglesas ${ }^{48}$. Se trata de una guía de viaje con varios mapas, dirigido al incipiente turismo, que de una manera muy sistematizada brinda al lector todo tipo de información necesaria, como la conexión de barcos, hoteles, vestimenta, médicos, excursiones, etc. 
A principios del siglo $X X$ aparecieron además dos obras que, a su descripción del viaje, añadieron mucho material visual en forma de fotografías. Se trata, por un lado, de la obra de Moritz Edenfeld sobre su viaje a las Islas Canarias y Madeira ${ }^{49}$, en la que describe este periplo con un cierto toque romántico. La otra publicación es un trabajo de Leo Woerl sobre su viaje a la misma región unos años más tarde ${ }^{50}$. Esta última, además de estar orientada como guía de viaje, tampoco contiene información de carácter científico, por lo que se puede entender más bien como un elemento publicitario para la isla de Madeira, en la que, según él, cada vez se encontraban más alemanes.

Mientras hasta ahora solamente han sido presentados estudios científicos o narraciones de viaje redactadas por hombres, las primeras obras sobre Madeira que manifiestan la visión de esta isla por una mujer, y que han podido ser localizadas, provienen de los primeros años del siglo XX. En este sentido, se puede mencionar el breve relato de Katharina Pommer-Esche de $1902^{51}$, escrito con un ligero romanticismo y que repite los estereotipos atribuidos a esta isla. Además, también se puede entender como propaganda de la isla, ya que en diferentes momentos lamenta que Madeira sea tan poco visitada por alemanes, que parecía más una colonia inglesa, y que para que esto no continuara siendo así, quería promover abiertamente la constitución de una sociedad alemana en Madeira ${ }^{52}$.

De similar estilo romántico, aunque no con esta intención explicita, presentada en forma de cartas de viaje a su familia y enriquecida con diversos poemas, es la obra de Anna Werner, publicada unos años más tarde ${ }^{53}$. Aunque estas dos muestras no serían lo suficientemente significativas como para poder deducir conclusiones sobre las diferencias en la obra en función de si el autor es hombre o mujer, al menos aquí se puede advertir un matiz más romántico, quizás poético $y$, en todo caso, divulgativo.

\section{ANÁLISIS}

\section{Visión de Madeira}

Se puede constatar que, en general, tanto las primeras impresiones de la isla de Madeira como las reflexiones hechas tras una estancia más larga, resultaron similares en las obras analizadas.

A Karl von Fritsch, por ejemplo, le encantaban los paseos por la ciudad y la abundante vegetación de Funchal, ya que "cada paso ofrecía nuevas bellezas de la vegetación de sur" ${ }^{44}$, pero aún se sintió mejor durante los calurosos meses de verano en la región montañosa de los alrededores de Santo da Serra (localidad también conocida como San António da Serra), desde donde realizaria pequeñas excursiones de carácter geológico $a$, entre otros lugares, Santa Cruz, Machico y Caniçal, y Porto da Cruz, los miradores del puerto de Lameiros así como al Pico Ruivo. Notablemente impresionado por los profundos valles, con sus cascadas y su maravilloso paisaje, lamentaba, no obstante, la predominancia inglesa en muchos aspectos, tales como la moneda, el comercio y el tráfico, a pesar de que la isla pertenecía a Portuga ${ }^{55}$. La influencia anglosajona constituía un asunto que comentaban 0 incluso criticaban abiertamente muchos viajeros de habla alemana, y al que dedicaban más o menos espacio en sus descripciones. Sivers nos informa que en las tiendas había sobre todo mercancía inglesa ${ }^{56}$, mientras Schultze comenta que los ingleses no aprendian portugués y además criticaba su falta de integración por mantener intactas sus costumbres, ya que su orgullo nacional no les permitía adaptarse a lo ajeno ${ }^{57}$. Todavía en el año 1885, en su guía de Madeira, Langerhans dice que se podía considerar como una colonia inglesa, y destaca que los ingleses no se mezclaban con la población, ni socialmente ni por matrimonio, como por ejemplo era el caso de los españoles en Tenerife ${ }^{58}$. Finalmente, como hemos indicado anteriormente, Katharina Pommer-Esche animaba a los alemanes a establecerse en la isla a fin de contrarrestar el predominio británico.

En la obra de Hermann Schacht se encuentran los mismos comentarios sobre la impresionante vegetación alrededor de Funchal, así como el predominio anglosajón en lo que se refiere a hoteles, comida, médicos, mercancía ofrecida en las tiendas, prensa, etc. ${ }^{59}$. Además, el autor describe la vida allí como sencilla y uniforme, ya que no había ni teatro ni conciertos y, como diversión, sólo se ofrecía una marcha militar en los paseos los domingos y festivos ${ }^{60}$. Esta falta de diversión pública, como la que podían brindar los teatros ${ }^{61}$, museos o galerías de arte, parece haber sido un tema a destacar para más viajeros ${ }^{62}$. 
Lo que Trogher apreciaba de la isla era la suavidad de su clima, que contribuía a mantener la salud y a llevar una vida sana y sencilla, la tranquilidad no perturbada por nada, y el olor de su vegetación. Con respecto a Funchal describe las casas limpias, con bonitos jardines, llenos de flores ${ }^{63}$, asi como las calles empinadas, muy bien pavimentadas con tablas de basalto, que podian resultar muy duras para andar, e incluso causar dolor al forastero ${ }^{64}$. En estas destaca además la falta de polvo en las calles, tan dañino para la salud ${ }^{65}$, un hecho que también otros viajeros han comentado muy positivamente ${ }^{66}$. Respecto a la oferta de fruta, comenta que Madeira reúne la ventaja de lo exótico combinada con lo europeo, sin que la gente se esforzara por mejorar la agricultura en este sentido ${ }^{67}$. La falta de dedicación a la mejora de la agricultura, es decir, una elaboración y sistematización, como pueden suponer el cambio de siembra o mejores instrumentos de labranza, constituye una crítica que se repite en muchas ocasiones ${ }^{68}$.

Mientras sobre el valle de Funchal, con su impresionante vegetación y muchas veces descrito como romántico, abundan las alabanzas, sobre la ciudad en sí se encuentran muy distintos comentarios. A Langerhans le parecía que nada en la ciudad era particularmente atractivo, pero aún así le causaba una impresión general muy agradable, destacando su nivel de limpieza, mayor que en otras ciudades de los puertos del sur. También a él las calles de basalto le resultaban incómodas, aunque prácticas contra la lluvia ${ }^{69}$. Sivers opina que la ciudad no era una de las más tentadoras ${ }^{70}$ y que no había mucho que mereciera la pena ver ${ }^{71}$. Parece que en estos aspectos no hubo mucha evolución a lo largo del tiempo, porque todavía en 1906 Stenzel consideraba que, como ciudad, Funchal ofrecía poco pero, en contraposición, el viajero sería plenamente recompensado por las afueras de la capital72. Lo que sí le parecía digno de mención eran los jardines de algunas de las villas y hoteles ingleses decorados con palmeras y magnolias ${ }^{73}$.

Un aspecto negativo comentado por diversos viajeros era la gran cantidad de mendigos que pululaban por la ciudad. Asimismo, era criticado el gran contraste social entre los pobres y los nobles de Madeira, así como la predominancia de los curas en la vida social de la población ${ }^{74}$. Sin embargo, a pesar de estas críticas, todos parecen haberse llevado una buena impresión de la isla, sobre todo de las excursiones que hicieron por la misma. Esto se debía, en gran parte, al clima suave e incomparable, y a la consecuencia de ello, es decir, la vegetación, los jardines, las frutas, etc., sobre lo que se encuentran todo tipo de apasionadas descripciones.

\section{Comentarios sobre la población}

Al margen de aquellos que han visitado Madeira con un determinado objetivo científico, o que no han incluido este aspecto social en su trabajo, en la mayoria de las publicaciones se encuentra un espacio dedicado a la descripción de la población, sea como un capítulo completo o bien como meros comentarios dentro de su narración de viaje, sea con un mayor interés por esta faceta de la realidad ajena, o de manera más superficial. Estas descripciones son, en unos casos, más objetivas, con una explicación de los hechos encontrados y los datos estadísticos ofrecidos; en algunos, se limitan a destacar las curiosidades vistas; y en otros, mantienen más un enfoque romántico, es decir, ensalzando lo auténtico y original de la vida sencilla hallada allí. Ciertas descripciones suscitan la impresión de que simplemente repiten algunos estereotipos de lo que suponen que sus lectores desean leer $y$, finalmente, también hay unas pocas que usan la descripción de lo ajeno para alabar su propia cultura de procedencia.

Sin embargo, no se ha encontrado ningún trabajo que se dedicase exclusivamente a la investigación de la sociedad de Madeira, como sucedía con sus caracteristicas geológicas o botánicas. Los posibles motivos para ello pueden ser que en Madeira destacaban más las particularidades de la ciencias naturales y que la sociedad encontrada no era lo suficientemente "ajena" como para que mereciera un estudio específico, o, sobre todo en los viajeros románticos, no se trataba de analizar la sociedad, sino emplearla sencillamente para reflejar su pintoresca visión del país.

En su estudio sobre el significado de Madeira para la curación de la tuberculosis, Schultze relata asimismo la vida social de la población indígena. Se trata de una descripción muy general, pero abarca muchos aspectos de esta faceta, basada en una dedicación más profunda a estas cuestiones $^{75}$. Así, por ejemplo, critica la influencia de la Iglesia en la sociedad, que se traduce para él en ignorancia, temor ante lo novedoso, superstición y bajo nivel de instrucción. Según él, era debido al escaso rendimiento de la agricultura y la ganadería así como al trato dispensado 
por los hacendados a los campesinos, lo que condujo a la ola de emigración. Resumiendo, dice que en aspectos climáticos podía elogiar a Madeira, sin incluir, no obstante, los elementos sociales, lo que hace que el viajero alemán se acordara "con melancolía de su gran y noble patria"76.

Asimismo, Langerhans concede espacio al factor social, dedicando todo un capítulo a la población ${ }^{77}$. Lo que destaca este autor, al igual que muchos de sus compañeros, es la rareza de la vestimenta observada alli, que describe como no muy pintoresca, y a la que pertenece un gorro de tela azul igual de raro y sin utilidad aparente ${ }^{78}$. La gente le parecía contenta aunque pobre, inconsciente, sin gran cultura, supersticiosa y con poca inclinación a la criminalidad. La dificultad de viajar, debido a la particular orografía montañosa del país, hace que haya poca movilidad entre la gente y que muchos nunca se aventuren a dejar su valle. Además, observa un bajo nivel de instrucción del pueblo, sin apenas creaciones literarias, salvo algunas traducciones del francés; asimismo comenta que no existía ningún librero en la isla $y$, finalmente, que en las clases altas el trato era muy cortés y se mantenía mucho la etiqueta.

Una descripción algo peyorativa de la población se encuentra en Trogher. Para él la poca población autóctona, aparte de los ingleses, alemanes, italianos y españoles que vivían allí, era de un aspecto más bien feo, con cierto toque árabe y ropa muy poco favorable o útil, sobre todo en lo que se refiere a los gorros ${ }^{79}$, cuestión esta que a tantos viajeros les parecía digna de mención. Respecto a la moral de los habitantes, le resultaba bien cuidada, pero remarcaba la falta de una vida espiritual o literaria, lo que tampoco le sorprendía debido a la pobreza de la isla ${ }^{80}$.

También Sivers brinda una corta, pero bastante negativa impresión de la población. En su opinión, se caracterizan por ser feos ${ }^{81}$ y por su pereza, muy extendida entre todos, un hecho que en esta región no podía ser disculpado por las altas temperaturas ${ }^{82}$.

En Stenzel, al contrario, se manifiesta más una visión romántica de la población. Según él, dentro de los hermosos e idílicos valles, donde la vida transcurre tranquilamente en paz y sin necesidades, el campesino ha permanecido inmune a las influencias negativas de la sociedad europea moderna, "de la caza loca por el dinero por la gente cegada"83.

\section{Comparación con las Islas Canarias}

Tal como se mencionó anteriormente, en muchos de estos relatos de viajes y estudios científicos surge inevitablemente la comparación con las Islas Canarias. En algunos casos ello se debe al hecho de que ambos archipiélagos fueron visitados en el mismo viaje, y para la elaboración del retrato de una realidad siempre servía como punto de referencia el de la anteriormente conocida. En otros casos llamaba la atención o bien los efectos del diferente tipo de dominio ejercido por los españoles en comparación con el de los portugueses, o bien el que en ambos casos se trataba de islas con caracteristicas particulares para la investigación científica. En otras palabras, la cercanía geográfica de estas islas, en combinación con sus diferencias históricas y de condición natural, ha motivado permanentemente esta comparación.

Este interés alemán por las Islas Canarias prosiguió también a finales del siglo XIX84. Tras los numerosos viajeros de nacionalidad inglesa y francesa que visitaron y exploraron las Islas Canarias desde comienzos del siglo XIX, estas lejanas y exóticas islas españolas llamaron cada vez más la atención también a los científicos de habla alemana. De esta manera, a partir de la segunda mitad del siglo XIX, Alemania se convertiria en la segunda nación por número de visitantes a este archipiélago, después de Gran Bretaña. Entre las distintas islas, Tenerife atrajo a más viajeros por su naturaleza exótica y, especialmente entre los de habla alemana, por la existencia del Teide, ya que la mayoría eran geólogos, botánicos y médicos. Otro aspecto a tomar en consideración es que la estancia de Alexander von Humboldt en esta isla en junio de 1799 y su entusiasta descripción posterior de la misma, sobre todo del Valle de La Orotava y su ascensión al Teide, animó a otros viajeros y naturalistas a emprender viajes de exploración por Tenerife. La segunda isla más visitada fue Gran Canaria, seguida por La Palma y, a continuación, Fuerteventura y Lanzarote, principalmente por viajeros con un marcado interés geológico, dado el carácter volcánico de las islas. El interés científico por estas islas también está vinculado al inicio del turismo y el significado de las Islas Canarias, al igual que Madeira, como balneario para afectados por ciertas enfermedades como la tuberculosis, en razón a sus benévolas condiciones climatológicas. Aparte de Humboldt destacan en este contexto los trabajos de Leopold von Buch, Ernst Heinrich Philipp August Haeckel, Karl Wilhelm 
Georg von Fritsch, Georg Hartung, Wilhelm Johann Reiß, Richard Greeff, Hermann Schacht, Friedrich Karl Noll, August Rothpletz y Hans Meyer ${ }^{85}$.

Algunos de estos viajeros conocían tanto el archipiélago canario como Madeira, lo que llevó a una comparación de estos lugares con arreglo a criterios distintos. A Hermann Schacht, por ejemplo, le interesaban las diferencias climáticas y de formación geológica, lo cual conducía a distintas condiciones para la vegetación. También Fritsch, cuando llega a Tenerife tras su estancia en Madeira, inmediatamente compara esta isla canaria con Madeira, con desventaja para la primera: según él, el entorno de la capital tinerfeña, los jardines así como los paseos públicos estaban dispuestos con menos arte y gusto que los de Funchal. No obstante, comenta que uno se sentía como en una ciudad europea, a pesar de ciertas apariencias extrañas ${ }^{86}$. El comercio, al contrario, le parecía más significativo que en Madeira, y el puerto, más animado. Además escribe que, debido a la frecuente comunicación marítima con los distintos lugares de Europa -aparte de una conexión regular interinsular-, las Islas Canarias le parecen más cercanas a su patria que Madeira. Asimismo, encontraba el sistema de riego mucho mejor organizado en Madeira que en Canarias, aunque la agricultura de ambas se situase a un mismo nivel de inferioridad. Con respecto a las comunicaciones, y debido al interés de numerosos viajeros por la isla, los desplazamientos por el interior de Madeira resultaban más fáciles y confortables. Finalmente, en lo que se refiere a la naturaleza, Fritsch encuentra mucho más impresionante la de Madeira que la de Canarias ${ }^{87}$.

Con esta pequeña introducción al tema de los viajeros de habla alemana a Madeira, se puede constatar que a partir de la segunda mitad del siglo XIX se inició una investigación científica sistemática y multidisciplinar de este archipiélago portugués ${ }^{88}$. Además de ello, se acrecentó paulatinamente la curiosidad por las islas por parte de los meros viajeros, lo que se refleja en el considerable aumento de publicaciones durante esta época, aparte del interés por Madeira como balneario. Su particular situación geográfica como isla contribuyó a suscitar el interés de científicos extranjeros así como de otros viajeros en general. Alli podian buscar lo autóctono, sin influencia ajena, ya fuera en alguna rama particular de las ciencias naturales, ya fuera en la vida y el paisaje en general. Por lo tanto, a lo largo del siglo XIX, pervive allí el espíritu romántico, sin menoscabo del positivismo de la actividad científica especializada. Lo auténticamente destacable en este contexto es la presencia de un considerable intercambio de información por parte de estos autores, que por lo general, al emprender la tarea de estudiar o describir la realidad de esta isla, se han dedicado a leer los trabajos realizados anteriormente.

\section{NOTAS}

* Trabajo realizado en el marco del proyecto de investigación del Ministerio de Ciencia e Innovación, número HUM2007-65125-C02-02, que dirige el Dr. Miguel Ángel Puig-Samper. La revisión bibliografíca, realizada en distintas bibliotecas de Berlín, ha sido financiada por la Deutsche Forschungsgemeinschaft (DFG). Una primera versión de este trabajo ha sido publicada como: Rebok, Sandra, "Aproximación al estudio de los viajeros de habla alemana por Madeira en el siglo XIX". As Ilhas e a Ciência. História da Ciência e das Técnicas. I
Seminário Internacional. Secretaria Regional do Turismo e Cultura. Madeira: Centro de Estudos de História do Atlântico, 2006, pp. 245-263.

1 Véase: Aragao, António, A Madeira vista por estrangeiros 1455-1700, Funchal: Secretaria Regional de Educación y Cultura, 1981. Una lista de publicaciones redactadas por extranjeros de diversas naciones sobre esta isla se encuentra al final de este libro. Los siguientes estudios ofrecen información general sobre la exploración científica por parte de los viajeros extranjeros en los últimos siglos: Vieira, Alberto, "A Madeira na rota da ciência e investigações cientifi-
Aceptado: 18 de septiembre de 2009 
cas". As Ilhas e a Ciência. História da Ciência e das Técnicas. I Seminário Internacional. Secretaria Regional do Turismo e Cultura. Madeira: Centro de Estudos de História do Atlântico, 2006 a, pp. 23-36; Vieira, Alberto, "As cidades Atlânticas e a história das ciências e das técnicas. Séculos XV-XX. As Ilhas e a Ciência. História da Ciência e das Técnicas. I Seminário Internacional. Secretaria Regional do Turismo e Cultura. Madeira: Centro de Estudos de História do Atlântico, 2006 b, pp. 109-129; Silva, Juan, "Explorações Botânicas na Madeira (Sécs. XVII-XIX). Uma abordagem Histórica". As Ilhas e a Ciência. História da Ciência e das Técnicas. I Seminário Internacional. Secretaria Regional do Turismo e Cultura. Madeira: Centro de Estudos de História do Atlântico, 2006, pp. 131-137. Una relación muy detallada de todos los viajeros que se dedicaron a lo largo de la historia al estudio naturalista de Madeira, así como sus publicaciones (incluidas las que salieron en revistas científicas) se encuentra en: Pão, Nélio, "A Madeira na rota da ciência e investigações cientificas. Listagem de personalidades que estudaram a história natural da Madeira (16011978)". As Ilhas e a Ciência. História da Ciência e das Técnicas. I Seminário Internacional. Secretaria Regional do Turismo e Cultura. Madeira: Centro de Estudos de História do Atlântico, 2006, pp. 37-108.

2 Debido a la situación política existente antes de la unificación de Alemania en el año 1871, es más preciso referirse a los viajeros de habla alemana, aunque por cuestiones de estilo, no se mantendrá esta distinción durante todo el texto. Además, de esta manera también se pueden incluir los trabajos realizados por austriacos y suizos, ya que entre este grupo de viajeros han sido leídos y recibidos de manera indistinta.

3 Osborne, John, Guide to the Madeiras, Azores, British and foreign West Indies, Mexico and northern SouthAmerica, compiled from documents specially furnished by the agents of the Royal Mail Steam Packet Company, and other authentic sources, London: Walton \& Mitchell, 1843, p. 18.

4 Entre las pocas personas que han trabajado sobre los viajeros alemanes en Madeira, destaca Eberhard Axel Wilhelm. Véanse, entre otros: Wilhelm, Eberhard Axel, Visitantes e Escritos Germânicos da Madeira 1815-1915, Funchal: Direcção Regional dos Asuntos Culturais, 1997; Wilhelm, Eberhard Axel, "Visitantes de lengua Alemã na Madeira (1815-1915)", Isleña 6, 1990, pp. 48-67; Wilhelm, Eberhard Axel, "As Experiência do Botânico Alemão Hermann Schacht (1855-1857): um ano e meio na Madeira", Islenha, 8, 1991, pp. 79-85; Wilhelm, Eberhard Axel, "A Madeira entre 1850 e 1900: Uma Estância de Tísicos Germânicos", Isleña, 13, 1993, pp. 116-121; Wilhelm, Eberhard Axel, "Os Madeirenses na Visãde Alguns Germânicos: o seu Aspecto e Carácter e a sua Maneira de Vivir I 1825 a 1860)", Xarabanda Revista, 7, 1995, pp. 2-13. Serrano Pinto, Manuel ha estudiado a fondo los trabajos de Georg Hartung, véase: Serrano Pinto, Manuel, "Georg Hartung and Charles Lyell and the Geology of the Madeira Island", As Ilhas e a Ciência. História da Ciência e das Técnicas, I Seminário Internacional. Secretaria Regional do Turismo e Cultura. Madeira: Centro de Estudos de História do Atlântico, 2006, pp. 403-416; Serrano Pinto Manuel, Annette Bouheiry, "The
German geologist Georg Hartung (1821-1891) and the geology of the Azores and Madeira islands", en P. N. Wyse Jackson (ed.), Four Centuries of Geological Travel: The Search for Knowledge on Foot, Bicycle, Sledge, and Camel, London: Geological Society, 2007, pp. 229-238.

5 La literatura mencionada, de ninguna manera, puede ser considerada como completa, ya que varios libros citados en estas mismas obras no han podido ser consultados; además faltaría una revisión sistemática de la prensa científica alemana de aquellos años en búsqueda de trabajos de viajeros de habla alemana por Madeira.

6 Mittermaier, Karl, Madeira und seine Bedeutung als Heilungsort. Nach vieljährigen Beobachtungen geschildert, Leipzig: F.C. Vogel, 1885.

7 Schultze, Rudolf, Die Insel Madeira: Aufenthalt der Kranken und Heilung der Tuberkulose daselbst. Nach dreijährigen Beobachtungen, Stuttgart: J.G. Cotta, 1864.

8 Entre otros sitios visitados menciona Cabo Girao, Santa Cruz, Machico, Santana, Pico Ruivo así como San Jorge.

9 Schultze, 1864, p. 16: En el original: "Von allem Kurorten aber, welche bis jetzt zu diesem Zwecke genauer durchforscht sind, ist es die Insel Madeira, besonders auf inr die Stadt Funchal, welche alle jene kosmetischen Verhältnisse darbietet, durch welche eine andauernde Heilung der Tuberkulose zumeist bedingt ist."

10 Ibidem, pp. 80-84. La bibliografía contiene casi todo las obras en inglés, con la excepción de estos trabajos en alemán: Heer, Oswald: Die periodische Erscheinung der Pflanzenwelt in Madeira, Zürich, 1851; Heer, Oswald, "Über den Naturcharakter und das geologische Alter von Madeira", Hel- 
vetia II (1) Zürich, 1852; Hochstetter, Ferdinand von, Madeira: Ein Vortrag. Vienna, 1861; Mittermeier, Karl, "Über Madeira", Zeitschrift der Gesellschaft der Ärzte zu Wien, suplemento n. ${ }^{\circ} 4$, 5, 1861; así como diversos trabajos de Hermann Schacht, Georg Hartung y de Wilhelm Reiss.

11 Ibidem, pp. 24-44.

12 Christmann, Ferdinand, Funchal auf Madeira und sein Clima, Altona: Hammerich \& Lesser, 1889.

13 Aquí se encuentran referencias a los trabajos Hermann Schacht; Karl Mittermaier y Paul Langerhans.

14 Christmann, 1889, p. 5.

15 Goldschmidt, Julius, Die Lepra auf Madeira, Leipzig: F. C. Vogel, 1891.

16 Richter, Johannes, Die klimatischen Grundlagen der landwirtschaftlichen Bodenkultur auf Madeira, tesis doctoral, Leipzig: Seele, 1909.

17 Schacht, Hermann, Madeira und Tenerife mit ihrer Vegetation: Ein Bericht an das Königlich Preussische Ministerian nebst einem Anhange: Eine kurze Schilderung meiner Reise und meines Aufenthaltes auf den Inseln, Berlin: G.W.F. Müller, 1859. Véase traducción al español: Batista Rodríguez, J., E. Gutiérrez Alonso y M. Sarmiento Pérez (ed.), Vegetación de Madeira y Tenerife (1859) de Hermann Schacht (introducción, traducción y notas). Las Palmas de Gran Canaria: Centro de la Cultura Popular Canaria (en prensa). Sobre este viajero váse además el trabajo de Wilhelm, 1991.

18 Schacht, 1859, p. 4.

19 Ibidem, p. 6.

20 Ibidem, p. 10.

21 Heer, Oswald, "Über die fossilen Pflanzen von St. Jorge in Madeira", Neue Denkschriften der allgemeinen Schweizerischen Gesellschaft für die gesamten Naturwissenschaften,
Zürich: Zürcher und Furrer, 1857, pp. 1-40.

22 Con la excepción de Georg Hartung, estas referencias se refieren sobre todo a trabajos ingleses como los de M. James Smith, Prof. Johnston, Charles Lyell, Eduard Forbes y Edward Bowdich, ya que en este momento todavía no existian muchos estudios en lengua alemana.

23 Hartung, Georg, Geologische Beschreibung der Inseln Madeira und Porto Santo, Leipzig: Wilhelm Engelmann, 1864. Sobre los trabajos de éste viajero véase Serrano Pinto, 2006 y Serrano Pinto/Bouheiry, 2007.

24 Los trabajos anteriores en los que se apoya eran, aparte de trabajos ingleses, los de Mittermaier y los de Wilhelm Reiss sobre sus investigaciones geológicas en Canarias, a efectos de comparación (Reiss, Wilhelm, Die Diabas- und Lavenformation der Insel Palma, Wiesbaden: C.W. Kreidel, 1861).

25 Greeff, Richard, Madeira und die Canarischen Inseln in naturwissenschaftlicher, besonders zoologischer Beziehung, Marburg: Univ.Schr., 1872.

26 lbidem, pp. 1-2.

27 Ibidem, pp. 20-33.

28 Greeff, Richard, Reise nach den Canarischen Inseln: London, Lissabon, Madeira, Tenerife, Gran Canaria, Lanzarote, Marokko, Spanien; mit populärnaturwiss. Schilderungen, Bonn: Cohen, 1868.

29 Fritsch, Karl Wilhelm Georg von, "Reisebilder von den Canarischen Inseln" (con 3 grabados de Gran Canaria, Hierro y Gomera), Mitteilungen aus der Justus Perthes' Geographischer Anstalt über wichtige neue Erforschungen auf dem Gesammtgebiete der Geographie, tomo supplementario $V$, n. ${ }^{\circ} 22$, Gotha, Justus Perthes, 1868, pp. 1-44.
30 Stübel, Alphons, "Briefliche Mitteilungen an Herrn H.B. Geinitz über die Geologie Madeiras (Beschreibung der Funchalbucht)", Neues Jahrbuch für Mineralogie, 1863, pp. 811-813; "Cabo Girao und Cama de Lobos auf Madeira", Globus, 7, 1865, pp. 332334.

31 Stübel, Alphons, Die Insel Madeira: photogr. Wiedergabe einer Reliefkarte zur Erläuterung des vulkan. Baues dieser Insel, editado por Walter Bergt, Leipzig: Weg in Komm., 1910.

32 Ibidem, p. V.

33 Véase cita en el prólogo de esta obra.

34 Trogher, Augustin, Briefe während einer Reise durch Istrien, Dalmatien, Albanien, Süd-Italien, Spanien, Portugal, Madeira und einem Theile der Westküste Afrika's, Triest: F. H. Schimpff's Buchhandlung, 1855.

35 Ibidem, pp. 165-175.

36 La palabra en alemán que emplea con gran frecuencia es "eigentümlich".

37 Trogher, 1855, p. 165. En el original: "... mit Recht ein Zufluchtsort, ein Heilort für Brustkranke genannt, aber nicht nur für Lungenkranke, sondern auch für Herzkranke, freilich nicht im physiologischen Sinne".

38 Sivers, Jegór von, Ueber Madeira und die Antillen nach Mittelamerika. Reisedenkwürdigkeiten u. Forschungen, Leipzig: Carl Fr. Fleischer, 1861.

39 Se trata de una bibliografía muy amplia respecto a América, pero sin incluir trabajos sobre Madeira.

40 Sivers, 1861, p. 15 (traducción propia).

41 Ibidem, p. 12: "Hier ist das Land, wo die Natur überreich die herrlichsten ihrer Gaben spendete und den Menschen zum Genusse auffordert. Hier ist es, wo der Mensch aller Sorgen vergessend, tief Athem holen kann aus reiner stärkenden Luft! Hier ist 
die Luft, die, ein erquickender Balsam, alle Wunden heilen mag (...)."

42 Stenzel, Johannes, Kreuz und quer auf Madeira und den Kanarischen Inseln, Berlin: Eduard Trewendt, 1906.

43 Ibidem, pp. 5-26.

44 Langerhans, Paul, Handbuch für Madeira, Berlin: Hirschwald, 1885.

45 A pesar de la aparente amplia difusión de esta obra entre los viajeros de la época, en ninguna de las bibliotecas consultadas se ha podido encontrar un ejemplar de la misma.

$46 \mathrm{Al}$ igual que sus antecesores, se refiere principalmente a estudios en lengua inglesa, pero como en este año ya existían numerosos trabajos en alemán, menciona distintos trabajos de los de Karl Mittermeier, Julius Goldschmidt, Oswald Heer, Richard Greeff, Rudolph Schultze.

47 Krohn, Ronald E. S., Führer durch Madeira, Berlin: Madeira-Actien-Gesellschaft, 1906.

48 Se refiere a Taylor, Ellen, Madeira and its scenery and how to see it: with letters of a year's residence, and list of the trees, flowers, fens and seaweeds, Londres: E. Stanford, 1882; y de nuevo, al ya mencionado manual de Madeira redactado por Yate Johnson.

49 Edenfeld, Moritz S, Eine Reise nach den Canarischen Inseln und Madeira, Straßburg: Singer, 1905. A Madeira dedica las páginas 60-73.

50 Woerl, Leo, Madeira und die Kanarischen Inseln in Wort und Bild, Leipzig: Woerl, 1914.

51 Pommer-Esche, Katharina von, Madeira die Wald-Insel, Berlin: H. Walther, 1902.

52 Ibidem, pp. 8-12.

53 Werner, Anna von, Reisebriefe: Madeira, Teneriffa, Gran Canaria, Kamerun, Dresden: Pierson, 1908.

54 Fritsch, 1868, p. 1.

55 Ibidem, p. 2.
56 Sivers, 1861, p. 11.

57 Schultze, 1864, p. 107.

58 Langerhans, 1885, p. 48.

59 Schacht, 1859, pp. 146-147.

60 Ibidem, p. 149.

61 Otros viajeros comentan que durante un tiempo sí hubo un teatro, aunque, al parecer, nada representativo.

62 Véase Langerhans 1885, p. 23.

63 Throgher, 1855, p. 166. En el original: "aus kleinen niedlichen, meist sehr reinen Häusern bestehend, die mit freundlichsten Gärtchen, voll der schönsten Blumen, untermischt sind".

64 Ibidem, p. 167. Véase también Christmann, 1889, p. 6.

65 Ibidem, p. 170.

66 Véase Christmann, 1889, p. 6.

67 Trogher, 1855, p. 170.

68 Véase Schacht, 1859, p. 15.

69 Langerhans, 1885, p. 4.

70 Sivers, 1861, p. 1.

71 lbidem, p. 10.

72 Stenzel, 1906, p. 6.

73 Ibidem, p. 10.

74 Sivers, 1861, pp. 11-12.

75 Schultze, 1864, pp. 87-107.

76 Ibidem, p. 91. En el original: "mit Wehmuth seines großen, edlen Vaterlandes".

77 Langerhans, 1885, pp. 40-48.

78 Ibidem, p. 41. Véase también Schultze, 1864, p. 103.

79 Trogher, 1855, p. 173. En el original: "(..) sind entschieden hässlich; ihr schwarzbrauner Teint, die arabische Conformation ihres Kopfes geben ihnen ein sehr unliebsames Aussehen; dazu kommt die Tracht, namentlich die der Weiber der ärmsten Volksclasse, die in einem weiten scharlachrothen Tuchmantel bestehend, der nothdürftig schlechte Lumpen und Fetzen bedeckt, ihnen eine grosse Ähnlichkeit mit unseren Seressanern verleiht; etwas besser sehen die Männer in ihren kurzen Leinwand- hosen und Hemdärmeln aus, komisch, und weder für Sonne, noch fûr Regen tauglich sind ihre Mützen von blauem Tuche (...). Gewöhnlich sind Volkstrachten, wenn auch nicht schön, doch wenigstens den Bedürfnissen des Klimas anpassend. Diese Tuchmützen sind aber das Unpraktischste, was ich in meinem Leben noch gesehen habe (...)".

80 lbidem, p. 175. En el orginal: "Was die Moralität der Einwohner anbelangt, so scheint sie, wenigstens dem äusseren Scheine nach zu urtheilen, und mehr steht einem Fremden nicht zu Gebote, streng gehütet. Von einem regen, geistigen Leben kann hier wohl keine Rede sein; bei einer so geringen Einwohnerzahl, wovon noch dazu die Meisten arm sind, kann man wohl keine literarisches Treiben oder auch nur den geringsten Buchhandel voraussetzen."

81 Sivers, 1861, p. 10. En el original: "Die Bewohner Madeira's zeigen sich durch ihre Hässlichkeit aus, es sind Neger, Mulatten und Portugiesen".

82 lbidem, p. 11.

83 Stenzel, 1906, p. 19.

84 Una recoplación de estos viajeros se encuentra en: Sarmiento Pérez, Marcos, Las islas Canarias en los textos alemanes (1494-1865), Las Palma de Canarias: Anroart Ediciones, SL, 2005; González Lemus, Nicolás, Viajeros, naturalistas y escritores de habla alemana en Canarias (100 años de historia, 1815-1915), Sevilla, Ediciones Baile del Sol, 2003; González Lemus, Nicolás, La mirada inacabada. Naturaleza y sociedad canaria en viajeros alemanes (desde Humboldt a Pannwitz), La Orotava, Tenerife; Ayuntamiento de la Villa de La Orotava, 2008; González Lemus, Nicolás (ed.), Recuerdos del Teide, Santa Cruz: Ediciones Idea, 2009. 
85 Puede hallarse más información sobre estos viajeros en: Rebok, Sandra, "España en la lente de los viajeros científicos alemanes durante el siglo XIX", en Llull, Revista de la Sociedad Española de Historia de las Ciencias y de las Técnicas, vol. 32, 2009, Zaragoza, pp. 135-152.

86 Fritsch, 1867, p. 3.

87 Fritsch, 1868, p. 2. En el original: "Madeira hat nicht wie jene Inseln die dürre Küstenregion mit ihren graugrünen Gewächsen von sonderbaren Formen, es besitzt nicht in seinen Höhen einen so ausgeprägten Hochgebirgs-Charakter wie Tenerife und etwa auch Palma, nicht jene Wüstennatur der beiden östlichen Canaren, inm fehlen die so eigenthümlichen weit ausgedehnten Kiefernwälder der Canarischen Gebirge; dafür sind auf den Canaren die Schönheiten der Natur weniger eng zusammengedrängt als auf Madeira und seltener sind dort als hier Zierpflanzen der Gärten der pflegenden Menschenhand entschlüpft, um doppelt üppig im Freien fortzukeimen (Fuchsia, die Hortensien, der Granatbusch \&c.). Durch reichere Bewässerung schon ist das an sich volkreichere Madeira mehr bebaut. Der zahlreiche Andrang der Fremden hat weiterhin nach Madeira einen Comfort und Reiseerleichterungen aller Art gebracht, die man auf den Canaren nicht wiederfindet."

88 Sin embargo, este estudio sólo ha podido dar unas pinceladas de este tema. Restaría el estudio de muchas cuestiones, como por ejemplo, analizar en mayor profundidad en qué consistía el interés particular por parte de Alemania, elaborar las características específicas de los viajeros de habla alemana, desentrañar las diferencias con franceses e ingle- ses, etc. Al margen de estos trabajos de análisis, se pueden estudiar más profundamente las obras particulares presentadas aquí.

\section{BIBLIOGRAFÍA}

Aragao, António (1981): A Madeira vista por estrangeiros 1455-1700, Funchal: Secretaria Regional de Educación y Cultura.

Batista Rodríguez, J.; E. Gutiérrez Alonso y M. Sarmiento Pérez (ed.): Vegetación de Madeira y Tenerife (1859) de Hermann Schacht (introducción, traducción y notas), Las Palmas de Gran Canaria: Centro de la Cultura Popular Canaria (en prensa).

Christmann, Ferdinand (1889): Funchal auf Madeira und sein Clima, Altona: Hammerich \& Lesser.

Edenfeld, Moritz S. (1905): Eine Reise nach den Canarischen Inseln und Madeira, Straßburg: Singer.

Fritsch, Karl Wilhelm Georg von (1868): "Reisebilder von den Canarischen Inseln" (con 3 grabados de Gran Canaria, Hierro y Gomera), Mitteilungen aus der Justus Perthes' Geographischer Anstalt über wichtige neue Erforschungen auf dem Gesammtgebiete der Geographie, tomo supplementario V, n. ${ }^{\circ} 22$, Gotha: Justus Perthes, pp. 1-44.

Goldschmidt, Julius (1891): Die Lepra auf Madeira, Leipzig: F.C. Vogel.

González Lemus, Nicolás (2003): Viajeros, naturalistas y escritores de habla alemana en Canarias (100 años de historia, 1815-1915), Sevilla, Ediciones Baile del Sol.

González Lemus, Nicolás (2008): La mirada inacabada. Naturaleza y sociedad canaria en viajeros alemanes (desde Humboldt a Pannwitz), La Orotava, Tenerife, Ayuntamiento de la Villa de La Orotava.
González Lemus, Nicolás (ed.) (2009): Recuerdos del Teide, Santa Cruz: Ediciones Idea.

Greeff, Richard (1868): Reise nach den Canarischen Inseln: London, Lissabon, Madeira, Tenerife, Gran Canaria, Lanzarote, Marokko, Spanien; mit populärnaturwiss. Schilderungen, Bonn: Cohen.

Greeff, Richard (1872): Madeira und die Canarischen Inseln in naturwissenschaftlicher, besonders zoologischer Beziehung, Marburg: Univ.-Schr.

Hartung, Georg (1864): Geologische Beschreibung der Inseln Madeira und Porto Santo, Leipzig: Wilhelm Engelmann.

Heer, Oswald (1857): "Über die fossilen Pflanzen von St. Jorge in Madeira", Neue Denkschriften der allgemeinen Schweizerischen Gesellschaft für die gesamten Naturwissenschaften, Zürich: Zürcher und Furrer, pp. 1-40.

Krohn, Ronald E. S. (1906): Führer durch Madeira, Berlin: Madeira-Actien-Gesellschaft.

Langerhans, Paul (1885): Handbuch für Madeira, Berlin: Hirschwald.

Mittermaier, Karl (1885): Madeira und seine Bedeutung als Heilort. Nach vieljährigen Beobachtungen geschildert, Leipzig: F.C. Vogel.

Osborne, John (1843): Guide to the Madeiras, Azores, British and foreign West Indies, Mexico and northern SouthAmerica, compiled from documents specially furnished by the agents of the Royal Mail Steam Packet Company, and other authentic sources, London: Walton \& Mitchell.

Pão, Nélio (2006): "A Madeira na rota da ciência e investigações cientificas. Listagem de personalidades que estudaram a história natural da Madeira (1601-1978)", As Ilhas e a Ciência. História da Ciência e das Técnicas, I Seminário Internacional. Secretaria 
Regional do Turismo e Cultura, Madeira: Centro de Estudos de História do Atlântico, pp. 37-108.

Pommer-Esche, Katharina von (1902): $M a-$ deira die Wald-Insel, Berlin: H. Walther.

Rebok, Sandra (2006): "Aproximación al estudio de los viajeros de habla alemana por Madeira en el siglo XIX", As Ilhas e a Ciência. História da Ciência e das Técnicas, I Seminário Internacional. Secretaria Regional do Turismo e Cultura, Madeira: Centro de Estudos de História do Atlântico, pp. 245-263.

Rebok, Sandra (2009): "España en la lente de los viajeros científicos alemanes durante el siglo XIX", en Llull, Revista de la Sociedad Española de Historia de las Ciencias y de las Técnicas, vol. 32, Zaragoza, pp. 135-152.

Richter, Johannes (1909): Die klimatischen Grundlagen der landwirtschaftlichen Bodenkultur auf Madeira, Tésis doctoral, Leipzig: Seele.

Sarmiento Pérez, Marcos (2005): Las islas Canarias en los textos alemanes (1494-1865), Las Palma de Canarias: Anroart Ediciones, SL.

Schacht, Hermann (1859): Madeira und Tenerife mit ihrer Vegetation: Ein Bericht an das Königlich Preussische Ministerium nebst einem Anhange: Eine kurze Schilderung meiner Reise und meines Aufenthaltes auf den Inseln, Berlin: G.W.F. Müller.

Schultze, Rudolf (1864): Die Insel Madeira: Aufenthalt der Kranken und Heilung der Tuberkulose daselbst. Nach dreijährigen Beobachtungen, Stuttgart: J.C. Cotta.

Silva, Juan (2006): "Explorações Botânicas na Madeira (Sécs. XVII-XIX).
Uma abordagem Histórica", As Ilhas e a Ciência. História da Ciência e das Técnicas, I Seminário Internacional. Secretaria Regional do Turismo e Cultura, Madeira: Centro de Estudos de História do Atlântico, pp. 131-137.

Sivers, Jegór von (1861): Ueber Madeira und die Antillen nach Mittelamerika. Reisedenkwürdigkeiten u. Forschungen, Leipzig: Carl Fr. Fleischer.

Serrano Pinto, Manuel (2006): "Georg Hartung and Charles Lyell and the Geology of the Madeira Island", As Ilhas e a Ciência. História da Ciência e das Técnicas, I Seminário Internacional. Secretaria Regional do Turismo e Cultura, Madeira: Centro de Estudos de História do Atlântico, pp. 403-416.

Serrano Pinto, Manuel y Bouheiry, Annette (2007): "The German geologist Georg Hartung (1821-1891) and the geology of the Azores and Madeira islands", en P. N. Wyse Jackson (ed.), Four Centuries of Geological Travel: The Search for Knowledge on Foot, Bicycle, Sledge, and Camel, London: Geological Society, pp. 229-238.

Stenzel, Johannes (1906): Kreuz und quer auf Madeira und den Kanarischen Inseln, Berlin: Eduard Trewendt.

Stübel, Alphons (1910): Die Insel Madeira: photographische Wiedergabe einer Reliefkarte zur Erläuterung des vulkanischen Baues dieser Insel, editado por Walter Bergt, Leipzig: Weg in Komm.

Trogher, Augustin (1855): Briefe während einer Reise durch Istrien, Dalmatien, Albanien, Süd-Italien, Spanien, Portugal, Madeira und einem Theile der Westküste Afrika's, Triest: F. H. Schimpff's Buchhandlung.
Werner, Anna von (1908): Reisebriefe: Madeira, Teneriffa, Gran Canaria, Kamerun, Dresden: Pierson.

Wilhelm, Eberhard Axel (1990): "Visitantes de lengua Alemãna Madeira (18151915)", Isleña, 6, pp. 48-67.

Wilhelm, Eberhard Axel (1991): "As Experiência do Botânico Alemão Hermann Schacht (1855-1857): um ano e meio na Madeira", Islenha, 8, pp. 79-85.

Wilhelm, Eberhard Axel (1993): "A Madeira entre 1850 e 1900: Uma Estância de Tísicos Germânicos", Isleña, 13, pp. 116-121.

Wilhelm, Eberhard Axel (1995): "Os Madeirenses na Visãde Alguns Germânicos: o seu Aspecto e Carácter e a sua Maneira de Vivir I 1825 a 1860)", Xarabanda Revista, 7, pp. 2-13.

Wilhelm, Eberhard Axel (1997): Visitantese Escritos Germânicos da Madeira 18151915, Funchal: Direcção Regional dos Asuntos Culturais.

Woerl, Leo (1914): Madeira und die Kanarischen Inseln in Wort und Bild, Leipzig: Woerl.

Vieira, Alberto (2006a): "A Madeira na rota da ciência e investigações cientificas", As Ilhas e a Ciência. História da Ciência e das Técnicas, I Seminário Internacional. Secretaria Regional do Turismo e Cultura. Madeira: Centro de Estudos de História do Atlântico, pp. 23-36.

Vieira, Alberto (2006b): "As cidades Atlânticas e a história das ciências e das técnicas. Séculos XV-XX", As Ilhas e a Ciência. História da Ciência e das Técnicas, I Seminário Internacional. Secretaria Regional do Turismo e Cultura, Madeira: Centro de Estudos de História do Atlântico, pp. 109-129. 